\title{
6
}

\section{ISE - Information Systems in Education}

\author{
Yaffa Gev \\ Department of Information Systems in Education, Ministry of Education, Jerusalem, Israel
}

\begin{abstract}
In order to cope with the complexity of Information Systems in Education, the Israeli government tackled the issue three levels on horizontally and vertically. The government established an information system on the head-quarter level, district and school level. Each one of them deals with the appropriate type of information according to the functional level.

The educational system is a complex system in which all levels must cope with the decision-making process. Therefore, information - which is a crucial resource in any system - is of supreme importance to the educational system.
\end{abstract}

Keyword Codes: H.4.2; K.3.1; K.4.1

Keywords: Information Systems; types of systems; Computers uses in Education; Public Policy Issues

\section{THE ISSUE}

Every organization requires complete, comprehensive up-to-date information. The information created in the organization and passing through it is varied and extensive, and is transferred among many systems running on a tight schedule.

As result inaccuracy and damage to data integrity may occur; the vast amount of data which is transferred is very difficult to monitor and data can get lost on its way from one subsystem or organization to another. Furthermore data can be understood differently in different systems and environments.

Failure to comply with schedules and deadlines arises because of delay in data-transfer, because the lack of know-how in compiling data into comprehensive, easy to get information. The main effect is reduced capacity for planning and decision-making and this brings us to a situation where planning based on accurate information is difficult to achieve.

In Israel, this issue takes the following shape: Israel's educational system (like any educational system) is characterized by a large number of users of different types, various use levels and needs government officials, political decision-makers, educational supervisors, school principals, teachers of all levels (some of them with very little knowledge handling information) parents, municipal officials etc. Each one of these uses information on various levels of detail and different extents of usage. A major problem is the "interpretation" each one of the above users gives to any data item. In many cases people relate differently to the same information just because of their diverse understanding of its meaning. 
Existing within the system are many subsystems which, while independent in function, have many common and overlapping areas of activity (such as the local authorities and the Ministry of Education). This causes duplication of data which usually leads to misinformation and disagreements. The system is extremely decentralized, with a wide geographical distribution and a large number of users. This affects the level of control and system supervision [1,2].

The advent of the information system, with the data access it provides, has had an immediate effect on every family and person in Israel, with long-term consequences. This can cause immediate critiques and disapprouval which can lead a lot of pressure on the educational system, much more than in other enviroment and systems.

In addition of functioning in a diffuse and large environment under constant pressure ISE in Israel suffers from constant shortages in teaching hours, resources, and budget - while, at the same time being under constant pressure to expand.

The solution is extensive computerization. Present-day advanced computer technologies enable the achievement of the following results:

Supply of all required data while maintaining data integrity, accuracy, and timeliness. Computers now are accessible to every one because of they small size, low cost and larger power. As data can be easily transferred so that users can achieve data integrity utilizing many data resources and compelling it to suit their special needs. Data can be used now as a basis for decision-making on all levels of organization.

As the awareness of information security increases, so does the need for tools and means of protection of data. Consequently there is also a demand for the implementation of the law for the protection of privacy. The new technology enables simultaneous access by many users in different systems. It enables combination of information from many different sources, with the required accessibility. It also enables examination of data and reaching decisions on the basis of decision-supporting ("what-if") techniques under shortage conditions. This brings us towards a more comprehensive ISE.

\section{THE ISE CONCEPT IN ISRAEL}

Information policy in education is derived from the policy of the educational system. The policy of Israel's educational system is based on the "state school" concept. In 1948, the Compulsory Education Law was passed, providing for free, compulsory education between the ages of 6 and 14 and stipulating joint management by the Government and the local authorities. In the 1970 s and 1980 s, the system tended toward decentralization of management (as a result of decisions made in order to increase efficiency). From the late 1980s until today, the trend toward separation continues. The strengthening of the community and local authority level has led to an increasing degree of autonomy in the schools.

Meanwhile, it was decided to extend free education up to grade 12 (age 18), and the consumption of educational and cultural services at the local level has increased. The guiding principle today is one of locally independent schools (autonomy) on one hand, and communication and integration on the other. Each subsystem within the overall educational system, including each school, is capable of autonomous action toward the collection and handling of data for its own needs.

At the same time, a system of standardization and procedures enables communication with other subsystems and integration of information. A standard manual (SMIS 1992) presents the SMIS framework in acordness with gravement standards. This manual is updated by the goverment form time to time. Thus the overall standardization procedure has been vastly inproved. 
The process has, however, not yet been completed. But schools can already "talk" with any other school and/or with any functional unit in the Ministry of Education or the local authority.

The ISE is based on: nationwide deployment of hardware and communications. A computer head-quarter center, based in Jerusalem, holds a 3090 I.B.M. system, Unissis and several DEC computers. The country is divided into seven districts each having its own computer center (DEC computer). The centers and schools and other sites there are hundreds of PC's. All the computer systems (not including schools) are interconnected and accessible on line. The intention is to put all systems on a wide area network which will be an overall educational network including schools and local authorities.

The policy of the Ministry of Education tends to lead to decentralization in information systems with central command and control. In order to do that, the Ministry, established a logistical system for data collection as close as possible to the generation of data. The system should enable: inter system (horizontal and vertical) transfer of information, provision of relevant, accurate, complete and available information at all user levels, standardization of information - determination of data standards for interfaces and processes, extensive use of technological innovations in the areas of communications and computerization, appropriate tools for each unit (subsystem); end users in schools, pedagogic centers and other educational entities, establishing two-way communication between them and the central information systems (Ministry of Education, authorities, research institutions, etc.).

The objective of ISE in Israel is to supply and support all users with accessible, reliable and updated data, and improved service to end users: staff, students and teachers. System effectiveness should be increased (reduction of the administrative burden; allocation of inputs to educational tasks). The recognition of the importance of information as a crucial resource led the government to establish a senior unit for information systems.

The structure of the Information System in Education $[4,5,6,7]$ rests on three main axes:

Teaching personel

Students

Institutional data and curricula

Data are stored at three information levels:

* Head quarter level - at which general data are stored pertaining to the entire population.

The more peripheral the user, the smaller the population covered and the greater the level of detail. At the same time, there are many areas in which the staff level - i.e., the Ministry maintains individual data for the needs of special projects of national importance.

* District level - storing information pertaining to the district and more extensive information concerning the school such as requirment study hours, subjects, etc.

Areas handled at these levels include:

Student achievements (aggregate, not for individual students)

Student dropout rates

Accidents, violence, insurance

Allocation of resources (standard per student)

Handling different population groups (gifted, special education, immigrants)

Planning of teaching personnel training

Curricula

Construction planning, registration areas, transportation 
* School level - detailed student data management, on the level of test and term mark; teacher data management, such as attendance and weekly lesson plans.

Systems operating within the school include:

SMIS (School Management Information System) - a tool for improving the effectiveness of the educational system in school; a standalone information system which enables data retrieval for decision making and management based on exceptional cases; a standardized tool enabling communication among all entities on a need-to-know basis. This latter tool allows internal control and supervision within the school through objective planning and performance control.

Self Evaluation - a tool for management of a pool of items and tests, test generation, and input of test results and data. This enables immediate assessment, from the teacher/coordinator/principal level (study of individual tests and student achievements) through the classroom level to the district and national level.

Telecommunications - for transfer of required report data to the various educational entities: Ministry of Education, owners of educational institutions, local authorities. It provides access to administrative databases (such as Director-General's circulars) and educational databases. It also has a capacity for technological communication among students and teachers, discussion groups, research projects, social education.

\section{CONCLUSION}

Information systems in education are operated according to the policy of the educational system, which determines the information management policy. The objective is to obtain the following:

Effective management through data and information

Planning and supervision

Compatibility of standardization

Availability of tools utilizing innovative computerization technologies

\section{REFERENCES}

1.Gev Yaffa, (1993) The "MANBAS" - A school Management Information System, G.U.I.D.E 34th Spring Conference

2. Feinmesser A. and Mendel S. (1989) Integrating school in education information systems: Pilot project conclusions (Hebrew) Jerusalem: The Israeli Ministry of Education and Culture.

3. SMIS Standards manual (1992) Jerusalem: the Israeli Ministry of Education and Culture (Hebrew) .

4. Telem M. (1987) The school computer administrator. Educational Technology.

5. Telem M. (1987) Conceptual and operational considerations for the planning and implementation of a pedagogical MIS on a national scale. Programmed learning and educational technology 24,187-193.

6. Ministry of Education, Culture and Sport (1992) "Information on Information Jerusalem.

7. Telem M.; Reuron G.; Epstein N. (1985) Pedagogical Management Information Systems in Israel. Report 1: Requirement Survey. 